\title{
Analysis of Risk Factors for Lymph Nodal Involvement in Early Stages of Rectal Cancer: When Can Local Excision Be Considered an Appropriate Treatment? Systematic Review and Meta-Analysis of the Literature
}

\author{
Alessandro Carrara, ${ }^{1}$ Daniela Mangiola, ${ }^{2}$ Riccardo Pertile, ${ }^{3}$ Alberta Ricci, ${ }^{4}$ Michele Motter, ${ }^{1}$ \\ Gianmarco Ghezzi, ${ }^{1}$ Orazio Zappalà, ${ }^{1}$ Gianni Ciaghi, ${ }^{1}$ and Giuseppe Tirone ${ }^{1}$
}

${ }^{1} 1$ st Division of General Surgery, S. Chiara Hospital, 38122 Trento, Italy

${ }^{2}$ Division of Medical Oncology and Palliative Medicine, Policlinic G. B. Rossi, 37134 Verona, Italy

${ }^{3}$ Department of Health, APSS, 38122 Trento, Italy

${ }^{4}$ Department of Obsterics and Gynecology, Policlinic G. B. Rossi, 37134 Verona, Italy

Correspondence should be addressed to Alessandro Carrara, alessandro.carrara@apss.tn.it

Received 15 March 2012; Revised 15 April 2012; Accepted 17 April 2012

Academic Editor: Manousos-Georgios Pramateftakis

Copyright ( 12012 Alessandro Carrara et al. This is an open access article distributed under the Creative Commons Attribution License, which permits unrestricted use, distribution, and reproduction in any medium, provided the original work is properly cited.

\begin{abstract}
Background. Over the past ten years oncological outcomes achieved by local excision techniques (LETs) as the sole treatment for early stages of rectal cancer (ESRC) have been often disappointing. The reasons for these poor results lie mostly in the high risk of the disease's diffusion to local-regional lymph nodes even in ESRC. Aims. This study aims to find the correct indications for LET in ESRC taking into consideration clinical-pathological features of tumours that may reduce the risk of lymph node metastasis to zero. Methods. Systematic literature review and meta-analysis of casistics of ESRC treated with total mesorectal excision with the aim of identifying risk factors for nodal involvement. Results. The risk of lymph node metastasis is higher in $\mathrm{G} \geq 2$ and $\mathrm{T} \geq 2$ tumours with lymphatic and/or vascular invasion. Other features which have not yet been sufficiently investigated include female gender, TSM stage $>1$, presence of tumour budding and/or perineural invasion. Conclusions. Results comparable to radical surgery can be achieved by LET only in patients with $T_{1} N_{0} G_{1}$ tumours with low-risk histological features, whereas deeper or more aggressive tumours should be addressed by radical surgery (RS).
\end{abstract}

\section{Introduction}

Colon and rectal cancers have many features in common, including risk factors, symptoms, and screening procedure; however, anatomical, clinical-pathological, and genetic diversities call for them to be considered as two different diseases often requiring different forms of treatment [1-4]. The oncological outcomes of rectal cancer surgery are usually worse than those of colon cancer; one reason for this is the higher local recurrence rate after curative resection [5]. The advent of total mesorectal excision (TME) as an addition to radical resection (RS) has strongly decreased the risk of local recurrence; in spite of this favourable outcome following RS, there is a high rate of severe complications and of abdominoperineal resections (APRs) with permanent colostomy. Local Excision (LE), more recently flanked by Transanal Endoscopic Microsurgery (TEM), has been proposed as an option for patients with early rectal carcinoma in whom radical surgery and its complications may be avoided, as well as for high-risk patients not suitable for administration of general anesthesia. Nevertheless, reported oncological outcomes following local excision techniques (LETs) in rectal cancer are often unsatisfactory. Local recurrence rates after LET for T1 and T2 tumours can range from $6.6 \%$ to $18 \%$ and from $17 \%$ to $67 \%[6-11]$, respectively [3-7]. Bentrem et al. found that patients with T1 rectal cancer treated by local excision have a threefold to fivefold higher risk of tumour recurrence than those treated by radical resection [12]. 
TABLE 1: Inclusion and exclusion criteria for study selection.

Inclusion criteria
(i) Casistics of rectal cancer T1/T2 treated by radical resection
with TME
Exclusion criteria
(i) Rectal cancer T3 or T4
(ii) Rectal cancer in IBD
(iii) Casistics of colon and rectal cancer together
(iv) Neoadjuvant therapies (radio/chemo)
(v) Radical resection of rectal cancer following LE/TEM
(vi) Radical resection of recurrent rectal cancer
(vii) Presence of distant metastasis (M1)
(viii) Studies focused only on selected histotypes (depressed
polyps, pedunculated polyps, etc.)
(ix) Studies focused only on selected lymph node involvements
(micrometastasis, lateral lymph node metastasis, etc.)

The reasons for these disappointing results can be attributed mostly to the high risk of the disease's diffusion to locoregional lymph nodes even in ESRC, for which LET cannot provide radical treatment. In this context it is clear that indications for the use of LET in ESRC should ideally consider all those clinical-pathological features that may reduce the risk of lymph node metastasis to zero. In this study risk factors for lymph node metastasis in ESRC were analysed through a systematic literature review together with a meta-analysis of the data retrieved.

\section{Materials and Methods}

2.1. Data Sources. Computerized search for mesh terms indicating risk factors for lymph node metastasis in rectal cancer up to December 2010 on Pubmed, Pubmed Central, OvidSP, BioMed Central, Chinal, Cochrane Library, Embase, SUMSearch, American College of Physicians (ACP) Journal Club, the most important Web Search Engines (Google, Google scholar, Yahoo, Lycos), grey literature, and references cited in the works selected.

A total of 136 studies were identified by the searches. By scanning titles and abstracts, 63 redundant publications, reviews, case reports, and editorials were excluded. After referring to full texts, 65 studies which did not satisfy the inclusion/exclusion criteria were removed from consideration. A total of 8 studies were left for analysis, involving a total of 1560 patients (Figure 1) [5, 13-19]. All 8 works were retrospective case studies of $\mathrm{T} 1 / \mathrm{T} 2$ primary rectal cancer treated with RS and TME, ranging from $2++$ to $2-$ according to the SIGN classification for grading evidence (Scottish Intercollegiate Guidelines Network) [13]. Unfortunately the objective of the work did not allow for other study designs apart from the analysis of retrospective studies. The inclusion and exclusion criteria are shown in Table 1.

Finally, the quantitative and qualitative data of 1560 patients were extracted from the works selected and reported in a cumulative data form. Variables collected for each

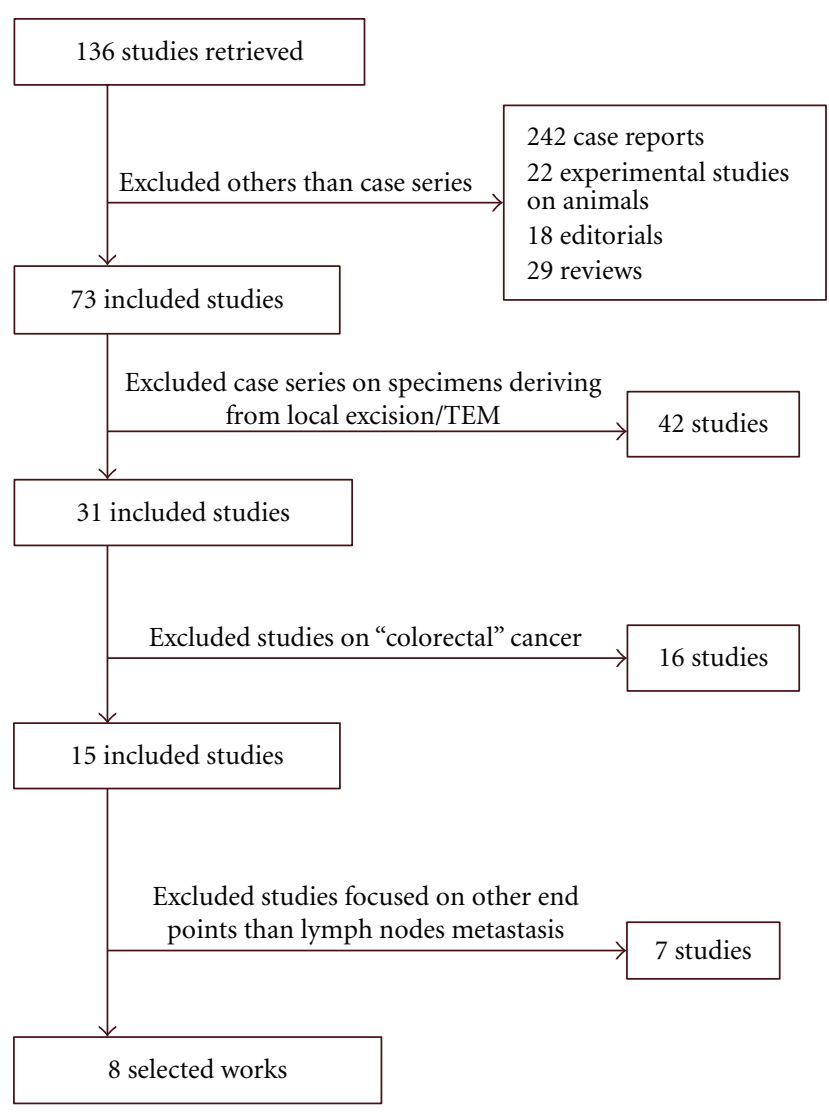

FIGURE 1: Quorum flowchart of the literature search.

patient included gender, age, tumour size, tumour grading, depth of tumour invasion, presence of lymphatic invasion, presence of vascular invasion, presence of perineural invasion, and tumour budding.

2.2. Statistical Analysis. Statistical analysis was carried out using Meta-DiSc (version 1.4). Study-specific ORs with corresponding 95\% confidence intervals (CIs) for different clinical and pathological features (dichotomous variables) versus loco-regional lymph node positivity/negativity were extracted. The overall effect was tested using $\chi^{2}$ with Yates correction or by Fisher's exact test (with significance being set at $P$-value $\leq 0.05)$. Meta-analysis was performed using fixedeffects (FEs) or random-effects (REs) models, depending on absence or presence of significant heterogeneity. Cochran-Q and Higgins $I^{2}$ statistics were used to check heterogeneity not only among studies but also between the subgroups included in this meta-analysis [14]. For the Cochran-Q statistic, $P$ value $\leq 0.10$ indicated statistically significant heterogeneity. We defined statistical significance as $P$-value $\leq 0.10$ rather than the conventional level of 0.05 because of the low power of this test [20]. $I^{2}$-values lie between $0 \%$ (no observed heterogeneity) and $100 \%$ (maximal heterogeneity); thus, an $I^{2}$ value greater than $50 \%$ may be considered to represent substantial heterogeneity [15]. In the absence of statistically significant heterogeneity, the fixed-effect method was used to 

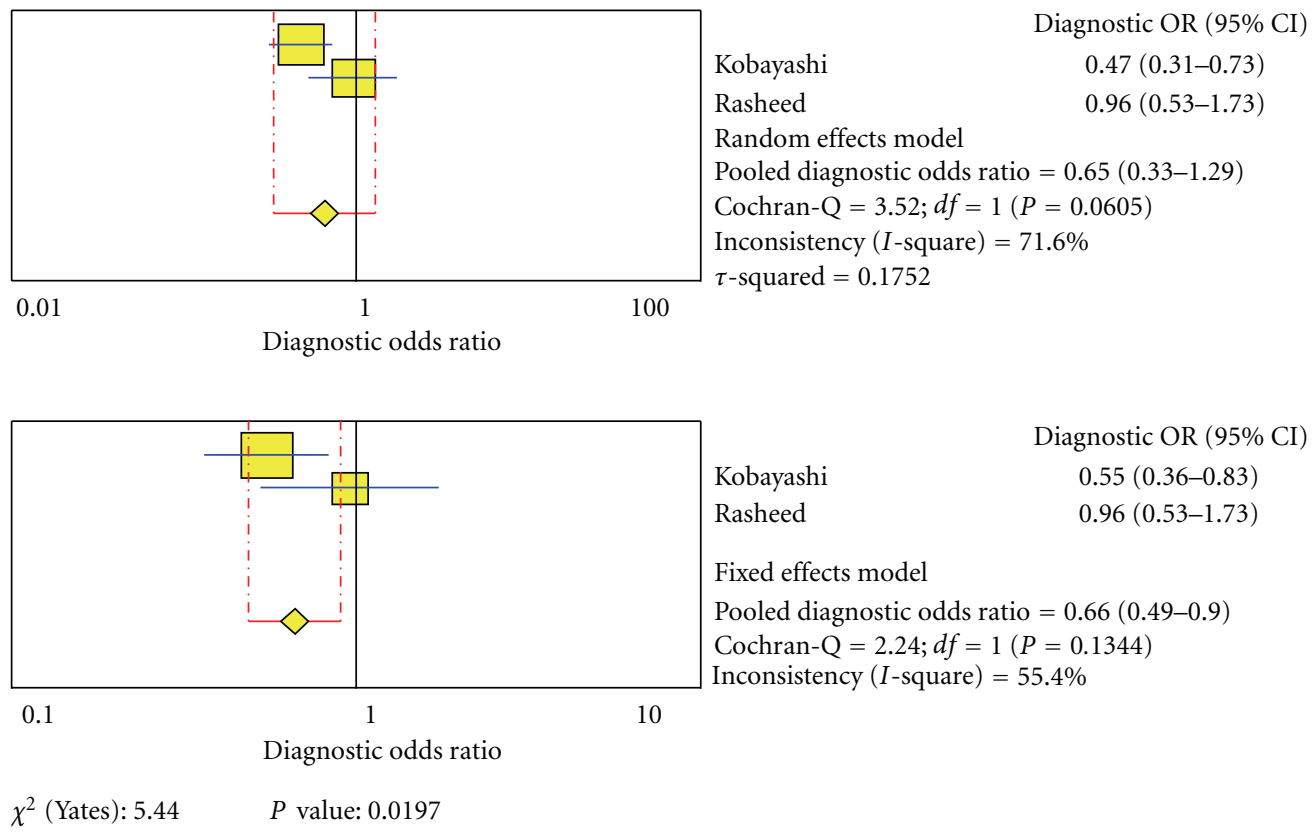

Kobayashi

Rasheed

Fixed effects model

Pooled diagnostic odds ratio $=0.66(0.49-0.9)$

Cochran- $\mathrm{Q}=2.24 ; d f=1(P=0.1344)$

Inconsistency $(I$-square $)=55.4 \%$

FIGURE 2: Risk of lymph node metastasis in males versus females.

combine the results. When heterogeneity was confirmed, the random-effect method was used.

\section{Results}

In our meta-analysis the rate of lymph node metastasis in ESRC is very high: about $10.53 \%$ for pT1 and $23.6 \%$ for pT2 cancers. Only two studies analysed gender as a prognostic factor for loco-regional lymph node metastasis (LNM), although the cumulative number of patients was quite substantial (870). Statistical heterogeneity between these studies was tested with Cochran-Q statistic $(P$-value $=0.13$, not significant) and with $I^{2}\left(I^{2}=55 \%\right.$, significant for presence of heterogeneity) (Figure 2). These contradictory results indicated that both fixed-and random-effect methods are useful to combine the results. In the first case the pooled OR for males vesus females in LNM was $0.66(0.49-0.90)$, with a $P$-value of 0.0197 , thus indicating a trend on the part of the female gender towards lymphatic spreading of the tumour. On the contrary, using a random-effect method, the pooled ORs for males versus females was $0.65(0.33-1.29)$, with a $P$-value of 0.17 ; even though the OR was not statistically significant, it displays a comparable trend. Moderate/high tumour grading and vascular invasion were analysed in five studies resulting in a strong association with the presence of LNM; pooled OR were 0.40 and 0.46 respectively, with $P$ values of 0.000 for both (Figures 4 and 5). A relevant association with LNM was also found for lymphatic invasion (pooled OR 0.26; $P$-value 0,000) (Figure 6). Depth of tumour invasion also proved to be an important prognostic factor for LNM. T1 versus T2 stages were examined in all selected studies with a much clearer trend of T2 stages towards nodal involvement (pooled OR 0.44; $P$-value 0.000) (Figure 3). Other tumour variables such as tumour size, tumour budding, perineural invasion, depth of tumour invasion within the submucosal layer ( $\mathrm{Sm} \mathrm{1,2,} \mathrm{and} \mathrm{3),} \mathrm{and} \mathrm{distance}$ from the dentate line were investigated either only in a single study or using heterogeneous parameters, thus preventing a reliable meta-analysis.

\section{Discussion}

Although colon cancer and rectal cancer share many features, there are important clinical-pathological and genetic differences between these two diseases, including in particular the tendency for rectal cancer, but not colon cancer, to recur locally; according to the majority of authors, this tendency towards local recurrence is a consequence of the tumour spreading through the lymph vessels. It has been clearly demonstrated that nodal involvement leads to an increased risk of local recurrence, overall survival, and disease-free survival $[16-19,21,22]$. In our meta-analysis the rate of lymph node metastasis in ESRC is very high: approximately $10.53 \%$ for $\mathrm{pT} 1$ and $23.6 \%$ for $\mathrm{pT} 2$ cancers. These outcomes stress how LET alone should not be considered as a radical oncological treatment in such a high subset of patients, unless future improvements in preoperative staging lead to the unequivocal identification of patients with nodal involvement. Unfortunately at present precise local tumour staging of rectal cancer is only possible after a surgical resection. The published literature shows that MR and US imaging, with an accuracy that does not exceed $70 \%$, are not reliable enough to identify nodal involvement [20,23-25]. A possible explanation for this trend is the frequent likelihood of metastasis of small lymph nodes (smaller than $5 \mathrm{~mm}$ ), which are difficult to detect even by highly experienced radiologists. In a recent study of 101 cases of rectal cancer $45.3 \%$ of the metastatic lymph nodes were Smaller than 5 


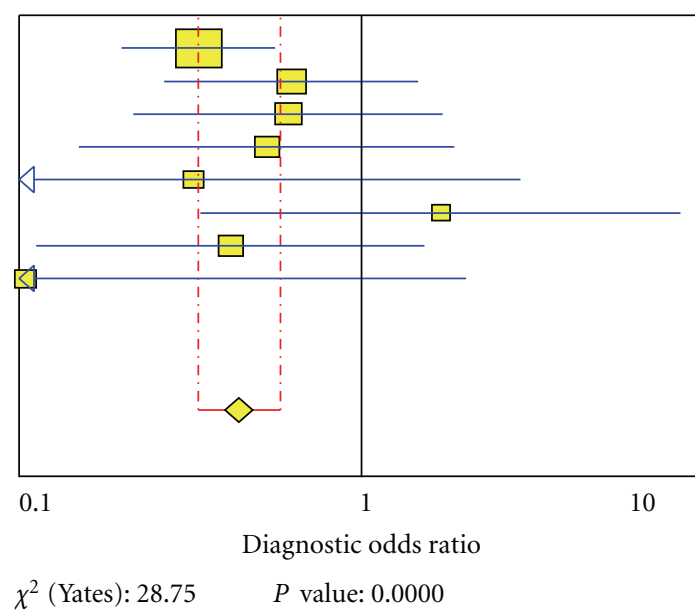

Kobayashi 2007

Rasheed 2008

Blumberg 1999

Brodsky 1992

Zenni 1998

Goldstein 1999

Huddy 1993

Saclarides 1994

Fixed effects mode

Pooled diagnostic odds ratio $=0.44(0.33-0.58)$

Cochran-Q $=5.89 ; d f=7(P=0.5524)$

Inconsistency $(I$-square $)=0 \%$

Figure 3: Risk of lymph node metastasis in T1 versus T2.

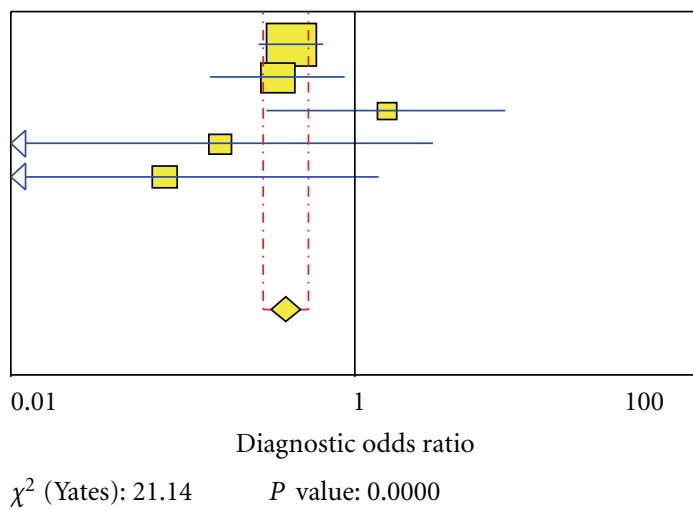

Kobayashi 2007

Rasheed 2008

Blumberg 1999

Brodsky 1992

Goldstein 1999

Fixed effects model

Pooled diagnostic odds ratio $=0.4(0.29-0.54)$

Cochran-Q $=4.47 ; d f=4(P=0.3467)$

Inconsistency $(I$-square $)=10.4 \%$

FIgURE 4: Risk of lymph node metastasis in G1 versus G2/G3.

millimetres in diameter; hence, a possibility of undetected nodal involvement even in T1 tumours does exist [23]. More recently MR supplemented with specific i.v. contrast medium (USPIO) was proposed by some authors as an alternative imaging technique offering high sensitivity and specificity in the identification of involved mesorectal lymph nodes. In the paper of Dow-Mu Koh based on 25 patients with rectal cancer the use of MR with USPIO resulted in an average sensitivity of $65 \%$; specificity, $93 \%$; positive predictive value, $43 \%$; negative predictive value, $97 \%$. The authors concluded that the use of MR with USPIO enhancement can achieve higher diagnostic specificity than but the same sensitivity as morphologic findings in pathologically matched mesorectal lymph nodes. Unfortunately given the shortage of trials addressing the outcomes of this amazing technique, its role in clinical practice still needs to be investigated in further studies. The sentinel lymph node technique has been more recently proposed by some authors as a means of evaluating loco-regional lymph node status; although interesting and promising, this technique is at present under development and has not yet been validated by scientific evidence [26, 27]. In the absence of a reliable technique to detect nodal involvement before surgery, research efforts are at present directed towards the identification of standard pathological variables capable of identifying tumours at risk of lymphatic spreading. Detecting a subset of patients who are likely to have LNM and who would possibly benefit from adjuvant therapies, abdominoperineal resection, or both would be of primary importance in the treatment of patients initially treated by local excision. Prior studies by other investigators have dealt with this issue through multivariate analysis of casistics of colorectal cancers operated on by radical surgery. Unfortunately these studies rarely discriminate colonic cancers from rectal cancers in their design. This differentiation is mandatory in our opinion, given the above-mentioned distinctions between these two diseases. To our knowledge, there are no systematic literature reviews with metaanalysis focused on risk factors for LNM exclusively in rectal cancer that deal with this problem. It is important to acknowledge the limitations of the present study: the retrospective nature and the restricted number of available studies investigating clinical-pathological tumour features could challenge our conclusions, but it must be said that the nature of this study per se does not permit any other kind of 


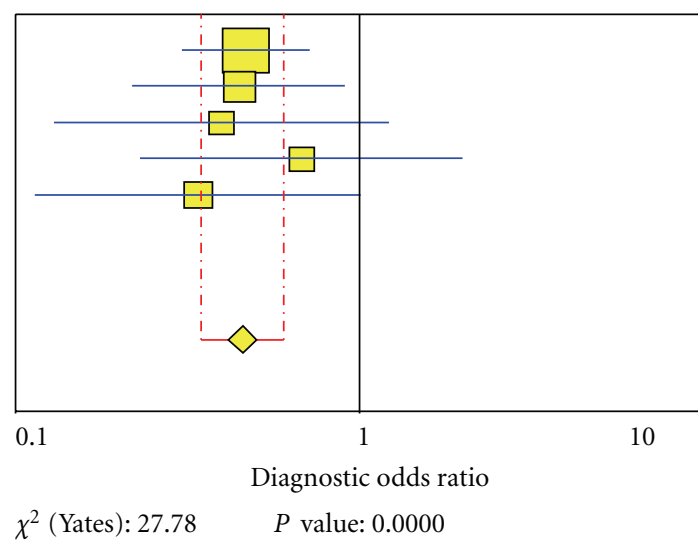

Kobayashi 2007

Rasheed 2008

Blumberg 1999

Brodsky 1992

Saclarides 1994

Fixed effects model

Pooled diagnostic odds ratio $=0.46(0.35-0.6)$

Cochran-Q $=0.87 ; d f=4(P=0.9282)$

Inconsistency $(I$-square $)=0 \%$

FIgURE 5: Risk of lymph node metastasis in Vascular Invasion.

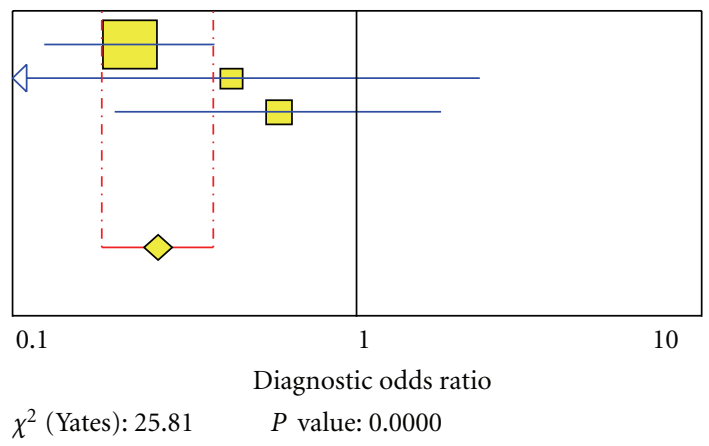

Kobayashi 2007

Blumberg 1999

Brodsky 1992

Fixed effects model

Pooled diagnostic odds ratio $=0.26(0.18-0.38)$

Cochran- $\mathrm{Q}=2.85 ; d f=2(P=0.2400)$

Inconsistency $(I$-square $)=29.9 \%$

FIGURE 6: Risk of lymph node metastasis in lymphatic invasion.

analysis than the retrospective investigation of rectal cancer specimens. Furthermore, in order to reduce selection biases, we followed rigorous exclusion criteria in the study selection. Studies which considered colon cancer and rectal cancer together were dismissed. We also excluded studies which did not discriminate between primary malignant tumours and recurrent ones, radiochemotherapy-treated cancer and cancer related to IBD. In addition we did not consider works focused on specific types of rectal cancer (i.e., sessile polyps, pedunculate polyps) or particular kinds of LNM (i.e., micrometastasis, lateral lymph node). Some of the findings that emerge from the present work call for a number of considerations: it is noteworthy that the female gender appears to be related to an increased risk of LNM (pooled OR with fixedeffect method equal to 0.66 ; $P$-value 0.019 ), but this finding was not significant using random-effect method, especially because it is difficult to identify heterogeneity in a so small number of studies. Nevertheless an explanation to this trend was attempted. Hormone receptors expressed on colorectal cancer (CRC) cells could play an important role in this context. The presence of estrogen receptors (ERs) has been clearly demonstrated in 70\% of CRCs [16]. Moreover, a number of other authors have shown that tamoxifen has a potent inhibitory action on metastatic cells from colo-rectal cancer in murine models [17]. It must be underlined that in our analysis this association is close to the inferior limits of the statistical significance and hampered by the fact that only two studies analyse this aspect (I2:55\%). New studies are needed to support this interesting finding. As previously shown in other studies on colo-rectal cancer, our data confirm that the risk of lymph node metastasis is increased in moderate or not differentiated tumours (grading 2-3) (pooled OR 0.40; $P$-value 0.000) [28], invasion of the muscular layer of the intestinal wall (pooled OR 0.44; $P$-value 0.000) [29], lymphatic invasion (pooled OR 0.26; $P$-value $0.000)$ [30, 31], and vascular invasion (pooled OR 0.46; $P$-value 0.000$)[32,33]$. The adequate number of studies included and the absence of heterogeneity between them mean that these outcomes have a high statistical significance. Other tumour variables such as tumour size, tumour budding, perineural invasion, depth of tumour invasion within the submucosal layer ( $\operatorname{Sm~1,~2,~and~3),~and~distance~from~the~}$ dentate line were investigated either only in a single study or using heterogeneous parameters, thus preventing a reliable meta-analysis. The following clinical-pathological features are worthy of further consideration.

4.1. Tumour Size. Tumour size can be an indicator of technical difficulty and can prevent the risk of postoperative complications (stenosis, leakage) and local recurrence due 
to the possibility of excision margin involvement. A tumour size of $3 \mathrm{~cm}$ involving $<40 \%$ of the rectal circumference has been taken as the upper limit by many authors. Some authors reported a trend toward higher local recurrence rates with tumour diameters $>3 \mathrm{~cm}$, although without a statistically significant difference [34]. Blumberg et al. [14] studied 3318 patients with intramural cancers (T1 or T2). Tumours classified as large $(>3 \mathrm{~cm})$ did not have an increased risk of lymph node metastasis when compared with small lesions $(\leq 3 \mathrm{~cm})$ with a $P$-value of 0.77 . Kobayashi et al. [5] analysed 567 consecutive patients who underwent radical resection for T1-T2 lower rectal cancer. The authors divided the lesions into two groups, smaller or equivalent to $2 \mathrm{~cm}$ and larger than $2 \mathrm{~cm}$, and did not find any statically significant difference in the incidence of lymph node metastasis between the two groups. Brodsky et al. [15] investigated 154 patients with pT1 or pT2 rectal cancer treated by radical resection. The authors found that increasing tumour diameter did not correlate with increasing incidence of LNM.

4.2. Distance from the Anal Verge. Distance from anal verge is of critical value not only for an increased risk of lymphatic spread but also because it can determine the surgical approach chosen. Steup et al. [35] studied 605 patients with rectal cancer. Of these, 44 were T1, 132 were T2, and 429 had a more invasive cancer. Steup did not study only patients with early rectal cancer, but from the analysis of the authors' findings it can be concluded that lesions of the lower third of the rectum have a higher incidence of lymph node metastasis. Nascimbeni et al. [31] identified an analysis cohort of 353 patients. Only patients with sessile T1 adenocarcinoma who underwent a colo-rectal resection were included in the study. The study results show that of 29 lesions in the lower third of the rectum, 10 (34\%) had lymph node metastasis; of the 54 patients with cancer in the middle third of the rectum, 6 (11\%) had lymph node invasion; finally, of the 36 patients with a tumour in the upper third of the rectum, $3(8 \%)$ had lymph node metastasis. The findings of the multivariate analysis ( $P$ 0.007) highlight that lesions of the lower third of the rectum have a higher risk of lymph node metastasis than the other rectal regions.

4.3. Sm 1-2-3. Some authors have analysed the relationship between the depth of invasion and the risk of lymph node metastasis according to Kudo's classification [36]. Nascimbeni et al. [31] studied histological specimens retrospectively from 353 patients undergoing colo-rectal resection for sessile T1 lesions. The authors reported a $1-3 \%$ risk of lymph node metastasis in $\mathrm{Sm} 1$ cancer and $8 \%$ in $\mathrm{Sm} 2$, while for Sm3 lesions the risk was 23\%. Thus they concluded that the invasion of the lower third of the submucosa (classified as "Sm3") is a significant predictor of lymph node metastasis. Rasheed et al. [13] analysed 313 patients with T1 and T2 colo-rectal cancer operated by radical resection with TME. The statistical analysis of this study failed to demonstrate a strong association between depth of tumour invasion and the presence of lymph node metastasis in T1 colo-rectal cancer. The author's conclusion however is that if depth of mucosal invasion is to be used as a guide to determine the likelihood of successful local curative surgery for rectal cancer, it must be used together with other prognostic indicators of success such as degree of tumour differentiation and evidence of vascular invasion. When the T1 tumour is superficial (Sm1 or Sm2) but one of these additional risk factors is present, the clinician should consider either more aggressive curative resectional surgery or the use of adjuvant oncological treatment in the form of chemotherapy or chemoradiotherapy.

4.4. Perineural Invasion. Perineural invasion refers to cancer spreading to the space surrounding a nerve. Perineural invasion is a well-known risk factor for nodal involvement in different types of tumours. With regard to colo-rectal cancer, Huh et al. [30] identified perineural invasion as the only significant independent factor predicting both overall and disease-free survival in patients with T1 and T2 colo-rectal cancer $(P=0.004)$. They found that although the incidence of perineural invasion was only $4.5 \%$, the odds ratio of lymph node metastasis increased 10 -fold for patients who had perineural invasion, as compared with those who did not. In their study, Saclarides et al. [19] found the same relationship between perineural invasion and nodal involvement as Huh.

4.5. Tumour Budding. Tumor budding is defined as an isolated cell or a small cluster of up to four carcinoma cells in the invasive front, and the presence of more than 10 budding foci when viewed at a 200 -fold magnification is considered positive for tumour budding, based on the data from Ueno et al. [37].

Several reports have suggested that tumour budding is probably the first histological event of invasion and metastasis in CRC. Okuyama et al. [38] reported that budding is a risk factor for lymph node metastasis in colo-rectal cancer, especially in the early stage. Homma et al. [39] examined tumour budding as a quantitative parameter to ascertain whether it could be used as an index for estimating the aggressiveness of early rectal cancers. He has found that a high tumour budding grade is a risk factor for lateral lymph node (LLN) metastasis and could be used as a criterion for LLN dissection. Goldstein and Hart [16] analysed 73 abdominal resection specimens and found a correlation between lymph node metastasis and tumour budding, even though with low statistical significance $(P$-value $<0.01)$. He suggests that pathologists should scrutinise the leading edge of locally excised cancer for foci of microacinar nest tumour budding or undifferentiated cells, because a patient is at increased risk of lymph node metastasis if any of these features is extensively present.

The results of the above studies, although not yet proven to be statistically significant, serve as an incentive to intensify the search of risk factors for lymph node invasion and the development of mathematical models to determine how much the risk increases in the case of coexistence of two or more of these features. Further studies are necessary on large casistics of ESRC with multivariate analysis of all the abovementioned clinical-pathological tumour features associated with lymph node invasion, in order to quantify the weight of each of these risk factors. A scoring system based on studies could be a good instrument to stratify patients into different 
risk classes and direct them towards the most appropriate treatment.

\section{Conclusions}

(i) Only the absence of each of the variables identified in this study (high-grade tumours, invasion of the muscular layer of the intestinal wall, lymphatic and vascular invasion) can justify LET as a radical treatment for rectal cancer.

(ii) If one of these risk factors is present, the risk of lymph node metastasis $\mathrm{N}+$ is real and the decision whether to refer the patient to an RS or an LE/MET in addition to radio-and/or chemotherapy should be taken in a multidisciplinary context, also taking into consideration patient characteristics and expectations.

(iii) A number of other tumour features are worthy of further investigation as potential risk factors for nodal involvement: gender, tumour size, tumour budding, distance from the dentate line, perineural invasion, and depth of submucosal invasion (Sm1, 2, and 3).

(iv) Further work is needed on large casistics of ESRC with multivariate analysis of all the above-mentioned risk factors in order to evaluate the weight of each of them and set up a scoring system to quantify the real risk of nodal involvement, stratify patients, and direct them to the most appropriate treatment.

\section{References}

[1] M. Frattini, D. Balestra, S. Suardi et al., "Different genetic features associated with colon and rectal carcinogenesis," Clinical Cancer Research, vol. 10, no. 12, pp. 4015-4021, 2004.

[2] K. Konishi, T. Fujii, N. Boku et al., "Clinicopathological differences between colonic and rectal carcinomas: are they based on the same mechanism of carcinogenesis?" Gut, vol. 45, no. 6, pp. 818-821, 1999.

[3] Y. Ikeda, M. Mori, K. Akagi et al., "Differences between features of adenoma in the rectum versus sigmoid colon," American Journal of Gastroenterology, vol. 95, no. 12, pp. 3620-3623, 2000.

[4] E. Kapiteijn, G. J. Liefers, L. C. Los et al., "Mechanisms of oncogenesis in colon versus rectal cancer," Journal of Pathology, vol. 195, no. 2, pp. 171-178, 2001.

[5] H. Kobayashi, H. Mochizuki, K. Sugihara et al., "Characteristics of recurrence and surveillance tools after curative resection for colorectal cancer: a multicenter study," Surgery, vol. 141, no. 1, pp. 67-75, 2007.

[6] A. Mellgren, P. Sirivongs, D. A. Rothenberger, R. D. Madoff, J. Garcia-Aguilar, and G. D. Steele, "Is local excision adequate therapy for early rectal cancer?" Diseases of the Colon and Rectum, vol. 43, no. 8, pp. 1064-1074, 2000.

[7] A. Chakravarti, C. C. Compton, P. C. Shellito et al., "Longterm follow-up of patients with rectal cancer managed by local excision with and without adjuvant irradiation," Annals of Surgery, vol. 230, no. 1, pp. 49-54, 1999.

[8] B. H. Endreseth, H. E. Myrvold, P. Romundstad, U. E. Hestvik, T. Bjerkeset, and A. Wibe, "Transanal excision vs. major surgery for T1 rectal cancer," Diseases of the Colon and Rectum, vol. 48, no. 7, pp. 1380-1388, 2005.
[9] J. Garcia-Aguilar, A. Mellgren, P. Sirivongs, D. Buie, R. D. Madoff, and D. A. Rothenberger, "Local excision of rectal cancer without adjuvant therapy: a word of caution," Annals of Surgery, vol. 231, no. 3, pp. 345-351, 2000.

[10] T. Hager, F. P. Gall, and P. Hermanek, "Local excision of cancer of the rectum," Diseases of the Colon and Rectum, vol. 26, no. 3, pp. 149-151, 1983.

[11] Y. N. You, N. N. Baxter, A. Stewart, and H. Nelson, "Is the increasing rate of local excision for stage I rectal cancer in the United States justified? A nationwide cohort study from the National Cancer Database," Annals of Surgery, vol. 245, no. 5, pp. 726-733, 2007.

[12] D. J. Bentrem, S. Okabe, W. D. Wong et al., "T1 adenocarcinoma of the rectum: transanal excision or radical surgery?" Annals of Surgery, vol. 242, no. 4, pp. 472-479, 2005.

[13] S. Rasheed, D. M. Bowley, O. Aziz et al., "Can depth of tumour invasion predict lymph node positivity in patients undergoing resection for early rectal cancer? A comparative study between T1 and T2 cancers," Colorectal Disease, vol. 10, no. 3, pp. 231$237,2008$.

[14] D. Blumberg, P. B. Paty, J. G. Guillem et al., "All patients with small intramural rectal cancers are at risk for lymph node metastasis," Diseases of the Colon and Rectum, vol. 42, no. 7, pp. 881-885, 1999.

[15] J. T. Brodsky, G. K. Richard, A. M. Cohen, and B. D. Minsky, "Variables correlated with the risk of lymph node metastasis in early rectal cancer," Cancer, vol. 69, no. 2, pp. 322-326, 1992.

[16] N. S. Goldstein and J. Hart, "Histologic features associated with lymph node metastasis in stage $\mathrm{T} 1$ and superficial T2 rectal adenocarcinomas in abdominoperineal resection specimens: Identifying a subset of patients for whom treatment with adjuvant therapy or completion abdominoperineal resection should be considered after local excision," American Journal of Clinical Pathology, vol. 111, no. 1, pp. 51-58, 1999.

[17] S. P. J. Huddy, E. M. Husband, M. G. Cook, N. M. Gibbs, C. G. Marks, and R. J. Heald, "Lymph node metastases in early rectal cancer," British Journal of Surgery, vol. 80, no. 11, pp. 1457-1458, 1993.

[18] G. C. Zenni, K. Abraham, F. J. Harford, D. M. Potocki, C. Herman, and P. B. Dobrin, "Characteristics of rectal carcinomas that predict the presence of lymph node metastases: implications for patient selection for local therapy," Journal of Surgical Oncology, vol. 67, no. 2, pp. 99-103, 1998.

[19] T. J. Saclarides, A. K. Bhattacharyya, C. Britton-Kuzel, D. Szeluga, and S. G. Economou, "Predicting lymph node metastases in rectal cancer," Diseases of the Colon and Rectum, vol. 37, no. 1, pp. 52-57, 1994.

[20] J. W. Huh, Y. A. Park, E. J. Jung, K. Y. Lee, and S. K. Sohn, "Accuracy of endorectal ultrasonography and computed tomography for restaging rectal cancer after preoperative chemoradiation," Journal of the American College of Surgeons, vol. 207, no. 1, pp. 7-12, 2008.

[21] Scottish Intercollegiate Guidelines Network, "SIGN 50: A guideline developer's handbook," 2008, http://www.sign.ac .uk/pdf/sign50.pdf.

[22] R. DerSimonian and N. Laird, "Meta-analysis in clinical trials," Controlled Clinical Trials, vol. 7, no. 3, pp. 177-188, 1986.

[23] H. Kim, J. S. Lim, J. Y. Choi et al., "Rectal cancer: comparison of accuracy of local-regional staging with two- and threedimensional preoperative 3-T MR imaging," Radiology, vol. 254, no. 2, pp. 485-492, 2010.

[24] S. A. Norton and M. G. Thomas, "Staging of rectosigmoid neoplasia with colonoscopic endoluminal ultrasonography," British Journal of Surgery, vol. 86, no. 7, pp. 942-946, 1999. 
[25] J. P. Heneghan, R. R. Salem, R. C. Lange, K. J. W. Taylor, and L. W. Hammers, "Transrectal sonography in staging rectal carcinoma: the role of gray- scale, color-flow, and Doppler imaging analysis," American Journal of Roentgenology, vol. 169, no. 5, pp. 1247-1252, 1997.

[26] R. A. Cahill, J. Leroy, and J. Marescaux, "Could lymphatic mapping and sentinel node biopsy provide oncological providence for local resectional techniques for colon cancer? A review of the literature," BMC Surgery, vol. 8, article no. 17, 2008.

[27] S. Noura, M. Ohue, Y. Seki et al., "Feasibility of a lateral region sentinel node biopsy of lower rectal cancer guided by indocyanine green using a near-infrared camera system," Annals of Surgical Oncology, vol. 17, no. 1, pp. 144-151, 2010.

[28] H. Y. Sung, W. K. Kang, S. W. Kim et al., "Risk factors for lymph node metastasis in patients with submucosal invasive colorectal carcinoma," Journal of the Korean Surgical Society, vol. 78, no. 4, pp. 207-212, 2010.

[29] K. S. H. Chok and W. L. Law, "Prognostic factors affecting survival and recurrence of patients with pT1 and pT2 colorectal cancer," World Journal of Surgery, vol. 31, no. 7, pp. 1485-1490, 2007.

[30] J. W. Huh, H. R. Kim, and Y. J. Kim, "Lymphovascular or perineural invasion may predict lymph node metastasis in patients with T1 and T2 colorectal cancer," Journal of Gastrointestinal Surgery, vol. 14, no. 7, pp. 1074-1080, 2010.

[31] R. Nascimbeni, L. J. Burgart, S. Nivatvongs, and D. R. Larson, "Risk of lymph node metastasis in T1 carcinoma of the colon and rectum," Diseases of the Colon and Rectum, vol. 45, no. 2, pp. 200-206, 2002.

[32] D. K. Sohn, H. J. Chang, J. W. Park et al., "Histopathological risk factors for lymph node metastasis in submucosal invasive colorectal carcinoma of pedunculated or semipedunculated type," Journal of Clinical Pathology, vol. 60, no. 8, pp. 912-915, 2007.

[33] Y. Kajiwara, H. Ueno, Y. Hashiguchi, H. Mochizuki, and K. Hase, "Risk factors of nodal involvement in T2 colorectal cancer," Diseases of the Colon and Rectum, vol. 53, no. 10, pp. 1393-1399, 2010.

[34] F. Bretagnol, E. Rullier, B. George, B. F. Warren, and N. J. Mortensen, "Local therapy for rectal cancer: still controversial?" Diseases of the Colon and Rectum, vol. 50, no. 4, pp. 523$533,2007$.

[35] W. H. Steup, Y. Moriya, and C. J. H. Van de Velde, "Patterns of lymphatic spread in rectal cancer. A topographical analysis on lymph node metastases," European Journal of Cancer, vol. 38, no. 7, pp. 911-918, 2002.

[36] "The Paris endoscopic classification of superficial neoplastic lesions: esophagus, stomach, and colon - November 30 to December 1, 2002," Gastrointestinal Endoscopy, vol. 58, no. 6, pp. S3-S43, 2003.

[37] H. Ueno, H. Mochizuki, Y. Hashiguchi et al., "Risk factors for an adverse outcome in early invasive colorectal carcinoma," Gastroenterology, vol. 127, no. 2, pp. 385-394, 2004.

[38] T. Okuyama, M. Oya, and H. Ishikawa, "Budding as a risk factor for lymph node metastasis in pT1 or pT2 welldifferentiated colorectal adenocarcinoma," Diseases of the Colon and Rectum, vol. 45, no. 5, pp. 628-634, 2002.

[39] Y. Homma, T. Hamano, Y. Otsuki, S. Shimizu, H. Kobayashi, and Y. Kobayashi, "Severe tumor budding is a risk factor for lateral lymph node metastasis in early rectal cancers," Journal of Surgical Oncology, vol. 102, no. 3, pp. 230-234, 2010. 


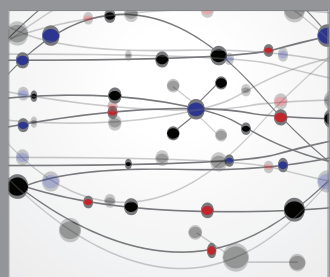

The Scientific World Journal
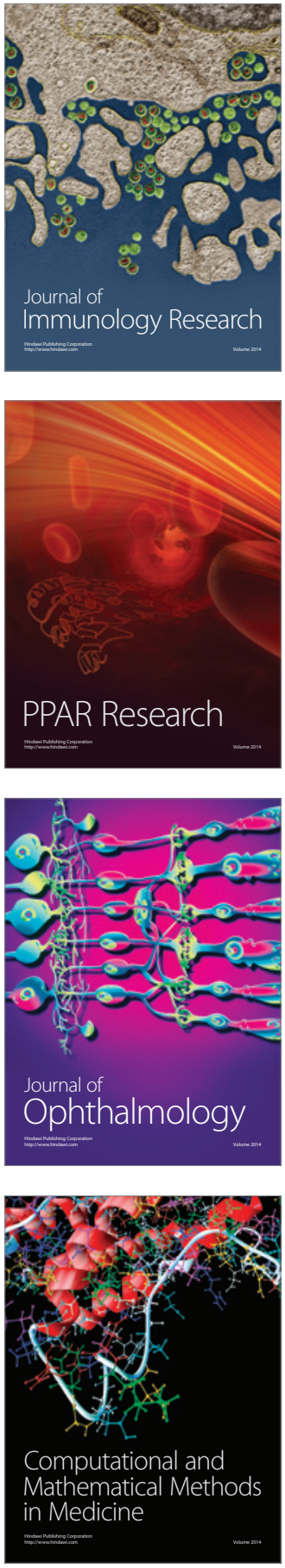

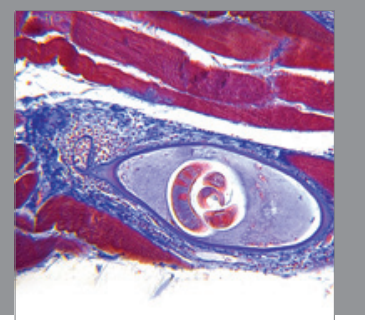

Gastroenterology

Research and Practice
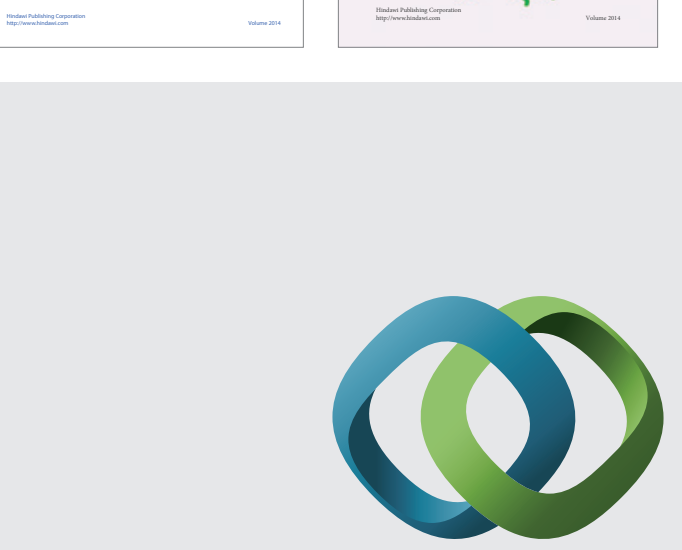

\section{Hindawi}

Submit your manuscripts at

http://www.hindawi.com
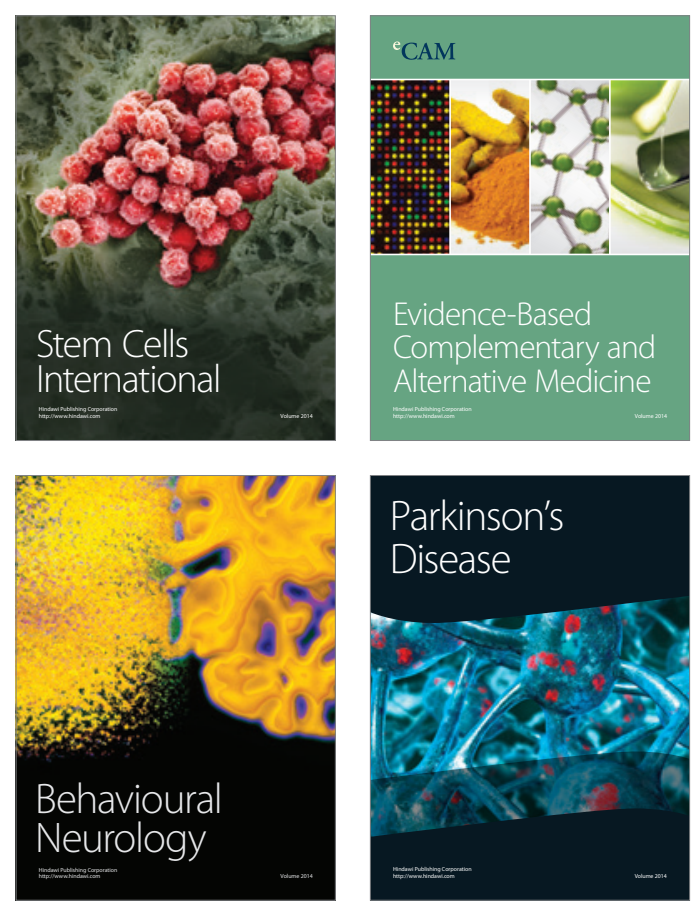

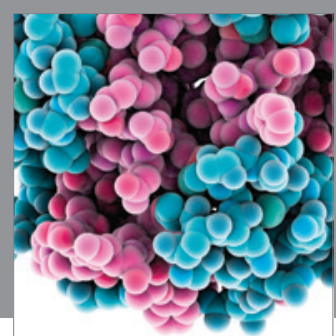

Journal of
Diabetes Research

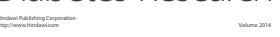

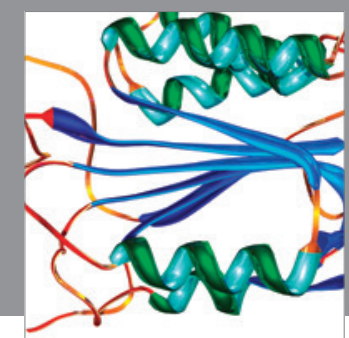

Disease Markers
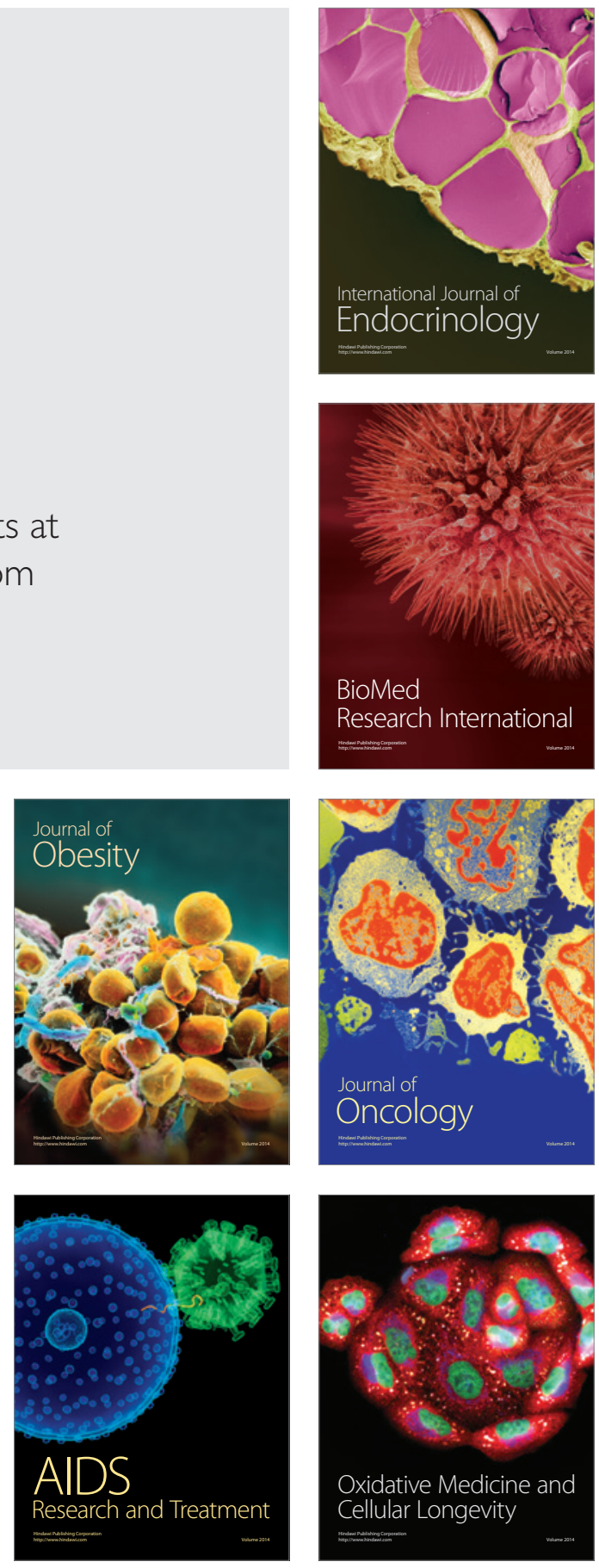